\title{
Tratamiento del Aborto
}

\author{
Dr. Gonzalo Echeverry P.
}

Vamos a considerar el tema del tratamiento del aborto en una forma global y resumida, con la idea de poder llevar al ánimo de los lectores una vista de conjunto que muestre una idea de lo que hoy en día se usa para tratar esta entidad, con algunas consideraciones sobre los fundamentos de estas terapéuticas, y sin entrar en detalles respecto a posología ni a casos particulares.

Para mejor comprensión, dividiremos el tema en los siguientes capítulos:

a) Tratamiento de la amenaza de aborto. b) Tratamiento del aborto inevitable. c) Tratamiento del aborto incompleto.

\section{a) TRATAMIENTO DE LA AMENAZA DE ABORTO}

Antes de entrar en el tema, debemos tener especial énfasis en que para el tratamiento de la amenaza de aborto se requiere como condición indispensable un diagnóstico correcto y exacto de la entidad, diagnóstico que deberá basarse en la existencia del embarazo y en la existencia de los signos ciínicos que muestran que la embarazada está amenazando abortar. Habrá que eliminar cuidadosamente cualquier causa de error en este diagnóstico, para no ir a tratar como amenaza de aborto un embarazo normal que no está amenazando abortar, o una hemorragia de cualquier otro origen, sea cervical, vaginal, urinario o rectal.

Sentado ésto, consideraremos el tratamiento de la amenaza de aborto dividido en dos grandes grupos: 1) El tratamiento causal o etiológico, y 2) El tratamiento sintomático.

\section{1) - TRATAMIENTO CAUSAL O ETIOLOGICO}

En la mayoría de los casos no es posible para el médico práctico determinar la etiología de la amenaza de aborto. Pero, cuando 
se presentan los casos en que esto es posible, se hará el tratamiento causal o etiológico, único verdaderamente racional e irrebatible en el estado actual de nuestros conocimientos. Vamos a considerar por separado las causas más frecuentemente demostrables de amenaza de aborto, y a dar los delineamientos generales de su tratamiento.

Hipotiroidismo. - Se corregirán los síntomas o signos evidentes de hipotiroidismo, o aún los signos clínicos presuntivos de deficiencia tiroidea, mediante la medicación de todos conocida. Hay quienes patrocinan el uso sistemático de la medicación tiroidea en los casos de amenaza de aborto aunque no se pueda poner en evidencia el hipotiroidismo, con la idea que de todas maneras vale la pena corregir una deficiencia aún no demostrada, por la influencia tan marcada que se atribuye a la función del tiroides en el metabolismo general de la embarazada y en el correcto desarrollo del producto de la concepción.

Naturalmente. es apenas razonable pensar que solo en los casos en que el hipotiroidismo es la verdadera causa determinante de la amenaza de aborto, ésta medicación tendrá un efecto cierto.

Función Luteal Deficiente. - Son pocos los casos en que, previamente a la iniciación del embarazo, se puede demostrar una deficięncia de la función luteal, basándose en los registros de temperatura basal y en los datos de la biopsia endometrial obtenida al final de la fase secretora o en la iniciación de las reglas.

Se trata en general de pacientes de ciclos cortos, en las que la curva de temperatura basal muestra una fase luteínica de poca duración, que no alcanza a producir en el endometrio las modificaciones progestacionales suficientes para la buena implantación y desarrollo del huevo, como puede comprobarse con la biopsia endometrial. En estas mujeres, si sobreviene la fecundación, se presume que el Cuerpo Lúteo gravídico sea también insuficiente para mantener las condiciones apropiadas de la caduca durante la iniciación de la gestación.

Son estos los casos en que está fundamentalmente indicada la terapia con progesterona, que obrará como medicación de supiencia de la función deficiente del cuerpo lúteo y logrará por 
consiguiente la formación de una caduca adecuada a la correcta nidación y desarrollo del huevo. Esta medicación debe continuarse por lo menos durante los tres primeros meses, hasta tanto que el sistema corio-placentario haya adquirido un desarrollo y una función suficientes para mantener un buen nivel progestínico y reemplazar así la secreción endocrina del cuerpo amarillo.

Es claro que en los casos en que por la historia de la paciente, o por cualquiera otra razón se sospecha la deficiencia luteínica cuando el embarazo ya está establecido y aún hay ya síntomas de amenaza de aborto, debe suministrarse la progesterona ya no como una profilaxis de la amenaza de aborto sino como verdadero tratamiento del aborto amenazante.

Más adelante nos referiremos a los sistemas propuestos para mejorar la función excretora de progestinas por parte del sistema corio-placentario, por medio de los estrógenos.

Incompetencia Cervical - El tratamiento del aborto amenazante debido a incompetencia cervical ha sido motivo de numerosísimas publicaciones, desde que Palmer llamó por primera vez la atención sobre este punto en 1948. Siend. la incompetencia cervical una de las causas de aborto habitual que más profusamente se han estudiado en los últimos 10 años, habrá que considerar el tratamiento profiláctico, es decir previo al embarazo, y el tratamiento que habrá de realizarse una vez constituída la gestación y presente la amenaza de aborto por esta razón.

Antes del embarazo, debemos distinguir la incompetencia cervical debida a laceraciones profundas, generalmente de origen obstétrico, la debida a intervenciones quirúrgicas anteriores (amputaciones del cuello). y la incontinencia cervical pura, por simple falta de oclusión adecuada del orificio interno.

En los casos de pacientes con historia de abortos repetidos, $y$ en las que se demuestra una incompetencia cervical por profundas laceraciones del cuello, el tratamiento se hará por medio de la cirugía reparadora conocida, es decir el avivamiento y sutura de las laceraciones. 
Los casos de amputaciones altas cervicales previas, en pacientes con historia de abortos, presentan mayores dificultades para el tratamiento quirúrgico previo al embarazo, por la falta misma del cuello que sirva como punto de apoyo al cirujano, pero de todas maneras deben tratar de corregirse. Estos casos tal vez reciban los mejores beneficios del tipo de operaciones de cerclaje que veremos más adelante.

El tratamiento, previo al embarazo, de la incompetencia cervical pura ha sido objeto de numerosos trabajos y técnicas operatorias propuestas, pero en sus delinearnientos generales se basa en las comunicaciones clásicas de Palmer (1948) y Lash \& Lash (1950), aunque ya desde 1941 A. Lash había propuesto la técnica del plegamiento de la pared anterior del segmento inferior y cuello uterinos.

Una vez hecho el diagnóstico por los métodos radiológicos y clínicos conocidos, se puede proceder a la operación electiva en cualquier época previa al nuevo embarazo, o a la operación inmediatamente después de presentarse un aborto. Este último sistema tiene más partidarios por el hecho de que se dice que es más fácil la realización de la intervención sobre un útero que aún no ha sufrido la involución post-aborto.

La técnica operatoria para estos casos es de dos tipos: en el uno, se busca estrechar el orificio interno del cuello mediante plegamiento de su pared anterior, o mediante la resección de una porción de forma oval en esta pared, para luego suturar y así estrechar el canal, o aún mediante la resección de una cuña completa de la cara anterior del segmento, que vaya hasta el orificio externo, suturando luego cuidadosamente para reconstituír así un canal más estrecho. De estos procedimientos ha conservado la predilección el de Palmer-Lash, o sea la resección de una porción oval de la cara anterior del segmento.

A los procedimientos anteriormente descritos se les ha hecho el reparo de que pueden ocasionar esterilidad secundaria por cierre cicatricial del cérvix, lo mismo que pueden presentar graves dehiscencias post-operatorias en las suturas, que en algunos casos, como uno referido por Page, condujeron por hemorragia a la histerectomía. 
Ermes Page, de la U. de California, propone una nueva técnica que considera exenta de los anteriores reparos. Se trata en resumen de disecar circularmente la mucosa alrededor del cuello uterino, para pasar por debajo de la fascia una sutura circular hecha con cinta de Catgut de $5 / 8$ de pulgada, y sostenida sobre el cilindro del cuello con tiras de oxicel embebido en benzoína y saturado con talco estéril, las cuales se usan para estimular la proliferación del tejido fibroblástico granulomatoso. El objetivo que el autor persigue con esta técnica es "crear una cicatriz fibrosa circular que resista la dilatación antes de que se inicie el trabajo". En los casos que llegan a la viabilidad, se permite el parto espontáneo por las vías naturales.

Nos hemos limitado a presentar las bases generales del tratamiento preconcepcional de la incompetencia cervical. No tenemos experiencia suficiente para pronunciarnos por uno u otro procedimiento, y personalmente solo podemos presentar un caso tratado por el sistema de Palmer-Lash, con excelente resultado, pues se obtuvo un niño vivo a término después de haberse presentado cuatro abortos alrededor de las 20 semanas de gestación, con los signos típicos de la incompetencia cervical.

El tratamiento de la incompetencia del cuello uterino durante el embarazo está basado en la operación propuesta por Shirokdar, de Calcuta, en 1955, y tiene como principio el cierre del orificio interno del cuello por medio de una sutura circular, submucosa, hecha con un material no absorbible.

La realización de esta operación tiene requisitos clínicos previos que no podemos pasar desapercibidos. En primer lugar, parece aceptado que la edad del embarazo adecuada para el tratamiento quirúrgico está entre las 14 y las 20 semanas de gestación. En segundo lugar, en los casos sospechosos debe practicarse examen con espéculo todas las semanas, a partir de la cuarta semana. Si en cualquier momento se observa que las membranas ovulares salen a través del cérvix, el caso debe considerarse como de incompetencia, y se tomarán las medidas adecuadas. Antes de la operación, la paciente debe permanecer en reposo absoluto por 48 horas, para descartar la posibilidad de un aborto en curso. Si 
después de este tiempo no se producen modificaciones apreciables, es decir que el aborto no avanza ni aparecen contracciones, se procederá entonces al tratamiento quirúrgico (Danforth).

La técnica operatoria se basa, como dijimos, en la de Shirodkar, es decir en un cerclaje lo más vecino posible al orificio interno del cuello, mediante material de sutura no reabsorbible. Las variantes propuestas son innumerables, pero la gran mayoría se refieren al uso de diferentes materiales de sutura, y a detalles técnicos que no se separan en nada fundamental de la técnica expuesta. Así se han usado para el cerclaje tiras de fascia lata, alambres de tantalum (desechados por el hecho de que cortan los tejidos), suturas con catgut cromado $\mathrm{N}^{\circ} 3$ en cinco o seis dobleces, lámina de catgut cromado, y cintas de diferentes materiales plásticos (Mersilene, Ethigraft) diseñadas especialmente para este efecto, y aún hilos gruesos de seda pasados a través de un tubo de polietileno. Actualmente se prefiere el uso de cintas plásticas.

A la intervención seguirá un período de reposo en cama por una semana, al mismo tiempo que la administración de opiáceos y progesterona. La paciente irá luego recobrando paulatinamente su actividad. En los casos buenos, el embarazo continuará hasta el término o cerca de él, y cuando comience el trabajo del parto, se podrá seccionar el material de cerclaje y permitir el parto espontáneo, caso en el cual para un embarazo subsiguiente volverá a presentarse el problema de la incompetencia y habrá que repetir el cerclaje o hacer el tratamiento quirúrgico antes de una nueva concepción. Si se desea conservar los beneficios del cerclaje, el parto se terminará por cesárea.

Deficiencia de Oxitocina. - Al continuar con el tratamiento causal de la amenaza de aborto, queremos hacer mención de un concepto emitido por el Prof. Edwin C. Hamblen en el curso de Endocrinología Ginecológica dictado en Bogotá en 1959. Según Hamblen, algunos abortos podrían ser debidos a que en la madre hay una cierta deficiencia de oxitocina, que daría por resultado un gradiente insuficiente de la contracción uterina fisiológica del embarazo, y por consiguiente un estímulo deficiente para mantener el orificio interno del cuello debidamente ocluído. El trata- 
miento de esta condición sería la administración de oxitocina en dosis muy pequeñas, que no alcancen a despertar contracciones uterinas evacuadoras, preferiblemente por vía nasal.

Respecto a este concepto, que nos parece altamente interesante a pesar de su apariencia un poco paradójica, no hemos podido conseguir ninguna otra fuente de información, y está por demás decir que carecemos en absoluto de experiencia en este sentido. No hemos querido dejar de mencionarlo por ser un aspecto completamente nuevo del problema, y que al menos en teoría parece ser muy lógico.

Histerasthenia Gravis. - Carl Javert define la Histerastenia Gravis como una hipertonicidad e irritabilidad aumentadas del útero grávido. Esta condición puede ser naturalmente causa de abortos que no tendrían otra explicación que las contracciones uterinas repetidas, que darían por resultado un "parto en miniatura". Puede apreciarse clínicamente por la palpación abdominal, que hace notar las contracciones uterinas intensas, y de duración que a veces llega a los dos minutos. El tratamiento de estas pacientes se referirá a tratar de sedar a la enferma y de suspender las contracciones uterinas mediante el reposo.

Fibrosis endometrial, Colagenosis corial. - Estas dos entidades histológicas se han descrito como causas de abortos. En relación con el tratamiento, se parte de la base de que son estas entidades manifestaciones de las enfermedades del colágeno, y por consiguiente su tratamiento se realizará con corticoestetroides su. prarrenales. Queremos anotar solamente que el uso de los corticosteroides en la embarazada es perfectamente permitido, y que al emplearlos después de la octava semana de gestación desaparece el peligro de malformaciones fetales o de masculinización de los fetos de sexo femenino.

Enfermedades Generales. - Es apenas obvio que cuando se presente una amenaza de aborto en una mujer que padece de una enfermedad infecciosa aguda, enfermedad general crónica, deficiencia nutricional, a- o hipovitaminosis, endocrinopatía, fragilidad capilar demostrada. etc., el tratamiento deberá dirigirse sin pérdida de tiempo a combatir la condición patológica presente, que puede determinar la interrupción del embarazo, máxime si se está ya en presencia de un estado de amenaza de aborto. 
Otras causas. - Las demás causas demostrables de amenaza de aborto, o de aborto mismo, están comprendidas dentro del capítulo de la patología ginecológica, endometrial, placentaria, funicular, o propiamente fetal. Las condiciones inherentes a mal desarrollo del huevo mismo solo serían susceptibles de corregir en teoría mejorando los factores genéticos o el factor endometrial, si aceptamos que puedan ser ocasionadas por mala preparación del endometrio. Es decir, que estos estados se salen del capítulo del tratamiento de la amenaza de aborto para pasar a ser parte del tratamiento pre-concepcional.

Igual cosa podemos decir de los factores puramente ginecológicos tales como malformaciones congénitas del útero, tumores endocavitarios o cervicales, etc., que deben ser corregidos antes del embarazo, para que sus resultados sean efectivos. En algunos casos de embarazos ya en curso con factores ginecológicos como los enunciados, podría intentarse la mejor nutrición del huevo mejorando las condiciones endometriales por medio de la progesterona.

\section{2) - TRATAMIENTO SINTOMATICO DE LA AMENAZA DE ABORTO}

Hemos visto cómo son pocos los casos en que se puede hacer el diagnóstico exacto de las causas de la amenaza de aborto, y por consiguiente un tratamiento causal definido. Quedamos entonces colocados ante la gestante que amenaza abortar, y en la que no podemos hallar la causa de esta amenaza.

Es en este grupo donde vamos a encontrar toda clase de tratamientos presentados por sus autores con estadísticas excesivamente halagueñas. que no siempre parecen ajustarse a la realidad.

Hertig afirma que el 50\% de los casos de amenaza de aborto mejoran por sí solos, y el embarazo continúa normalmente, aunque no se haga ninguna terapéutica. Si se acepta esta cifra, y la afirmación de que un $15 \%$ de los abortos se deben a factores ovulares, que de todas maneras conducirán al aborto, vendría a quedar solo un $35 \%$ de casos de amenaza de aborto que podrían beneficiarse del tratamiento (Hamblen). Ahora bien, cualquiera de los preconizadores de los distintos tratamientos propuestos, da 
un $70 \%$ a $80 \%$ de éxitos; pero si a esta cifra se le quita el $50 \%$ de que habla Hertig, en realidad el éxito solo se referirá al 20$30 \%$ restante.

Entonces la amenaza de aborto que vamos a tratar, cuando no conocemos su causa, será aquella en que, sin saberse por qué, se producen contracciones uterinas de mayor intensidad que las normales del embarazo que adquieren un gradiente del mismo tipo de las del parto, y que si persisten van a provocar el desprendimiento parcial o total del huevo, con borramiento y dilatación del cuello. Si se trata de un huevo normal y los desprendimientos producidos abarcan apenas zonas muy limitadas del trofoblasto, al cesar las contracciones uterinas, el embarazo podrá continuar. Si los desprendimientos son amplios o totales, el aborto se producirá irremisiblemente.

Para el tratamiento de la amenaza de aborto se han preconizado a través de toda la historia médica infinidad de conductas terapéuticas, las cuales han adolecido casi siempre, y aún en la época contemporánea, de un mayor o menor empirismo. Pasaremos una pequeña revista crítica a los tratamientos aún en uso.

Cloral, Opiáceos. - Desde hace muchos años se ha pretendido disminuír o suprimir la contractilidad uterina sobreexcitada por medio de la administración rectal de hidrato de cloral y de Láudano, sin que se haya podido demostrar jamás que esta medicación tenga ningún efecto frenador sobre el útero. Su utilidad estaría relacionada exclusivamente a su acción hipno-analgésica.

Lo mismo podemos decir de la administración parenteral de morfina o sus derivados o sucedáneos sintéticos, con el agravante de que la aplicación de estos sedantes enérgicos, que suelen tener un gran poder antiespasmódico, presenta el peligro de que, al continuar las contracciones uterinas hechas indoloras por su potente acción analgésica, se haga con más facilidad la dilatación del cuello y así se produzca más pronto la expulsión del huevo.

Glicerofosfato de Sodio. - Se ha insistido mucho por parte de los fabricantes, en la acción frenadora de la contractilidad uterina que tienen los glicerofosfatos de sodio administrados por vía 
endovenosa. Desgraciadamente, esta acción frenadora de los glicerofosfatos sobre el útero se hace clínicamente más aparente en las amenazas de parto prematuro por encima de las 25 semanas de embarazo, que en las amenazas de aborto.

Progesteronas. - Desde el momento mismo en que la progesterona fué aislada y se pudo obtener en los mercados, esta hormona ha estado en el primer plano de la terapia anti-abortiva. Con un criterio bastante simplista se pensó en un principio que si la función luteal tenía como fin primordial proteger el huevo, el tratamiento racional de la amenaza de aborto sería el suministro de la hormona del cuerpo amarillo en dosis que, con el correr de los días han ido aumentando hasta cifras de 250 miligramos diarios.

La indicación del uso de progesterona en la amenaza de aborto estaría respaldada por la acción frenadora de la contractilidad uterina que clásicamente se le ha atribuído a esta hormona, y por su acción estimulante muy definida de los cambios endometriales que han de constituír una caduca apropiada para la buena nidación y desarrollo del huevo. Además, ya vimos al hablar del tratamiento causal del aborto que en los casos de deficiencia demostrada de función del cuerpo lúteo, la progesterona pre y post concepcional es el tratamiento racional y electivo.

Por otra parte, se han realizado innumerables estudios de dosificación de pregnandiol urinario en los casos de amenaza de aborto, con el resultado de que en la mayoría de ellos no existen cifras bajas de pregnandiol, lo que quiere decir que no hay deficiencia progesterónica. También se ha podido comprobar plenamente que la administración de progesterona, cualquiera que sea la dosis, la vía, y el tipo de progesterona usados, no modifica los niveles de pregnandiol urinario, los cuales, cuando se hacen críticos, conducen irremediablemente al aborto.

Estas son las razones por las cuales en muchos medios la progesterona ha perdido su lugar de preeminencia en el tratamiento del aborto, y en algunos otros ha llegado hasta ser completamente proscrita mientras no se le demuestren indicaciones específicas. En otros medios, sigue en el primer plano de la terapia anti abortiva, aunque al analizar desprevenidamente la ind: 
cación se encuentre que su uso peca de cierto empirismo, y que es una droga que todos los días se está administrando en multitud de pacientes sin que al prescribirla se tenga como respaldo una base fisiopatológica precisa y racional.

No está por demás advertir que el uso de los progestínicos en la embarazada, así sean los nuevos y potentes derivados de la nor-testosterona y de la etisterona, está más o menos exento del riesgo que en un principio se les atribuyó de masculinización de los fetos de sexo femenino.

Estrógenos. - Teniendo en cuenta la poca firmeza que en ocasiones tiene la indicación de la progesterona en el aborto, se han buscado otros sistemas de tratamiento, de los cuales uno de los que despertaron mayor interés fué el de Smith y Smith, propuesto por primera vez hace 10 años. Los autores se basaron en que al administrar estrógenos a la embarazada en dosis crecientes y elevadas, éstos obrarían sobre la caduca en el sentido de darle una mayor vascularización y actividad circulatoria, lo cual traería como consecuencia un mejor funcionamiento del sistema corio-placentario, y una mayor producción de progestinas.

En apoyo de su teoría, los Smith encontraron que a medida que semanalmente aumentaban la administración de estrógenos a sus pacientes, los niveles de pregnandiol urinario subía en proporción al aumento de la dosis estrogénica, resultado que no pudo lograrse nunca con la progesterona. Emplearon en sus tratamientos el dietil-estil-bestrol por ser éste un estrógeno potente, de poco costo, y muy bien tolerado por las embarazadas.

Con estas bases, y unas cifras estadísticas de resultados clínicos de cerca de un $80 \%$ de éxitos en sus casos personales, el sistema parecía incombatible, y fue extraordinario el entusiasmo que en todos los medios despertó. Por desgracia, posteriores investigaciones llevadas a cabo en la Universidad de Duke pudieron comprobar que lo que la escuela de Boston informaba como niveles de pregnandiol urinario crecientes, eran simplemente dosificaciones de complejo de glucuronato de dietil-estil-bestrol y no del complejo de glucuronato de pregnandiol sódico, complejos que tienen reacciones químicas muy semejantes. En esta forma quedó destruído el pilar principal en que se apoyaba el tratamiento con dietil-estil-bestrol. 
Estrógenos - Progesterona. - Al no encontrarse una base completamente firme para el uso aislado de una de las dos hormonas, se ha propuesto usarlas combinadas para poder obtener de cada una de ellas los elementos que pueden ser útiles. Sería ésta la manera más lógica de usar la hormonoterapia en la amenaza de aborto, cuando no se tienen indicaciones precisas de su empleo.

Vitamina $E$ - Ya pasó hace años el auge del uso de los tocoferoles en la amenaza de aborto, y es hoy en día una terapia casi completamente descontinuada por no haber podido demostrar su utilidad.

Vitamina C., Vitamina K., Bioflavonoides. - El uso de estos trus medicamentos combinados ha sido ampliamente preconizado por Javert, de New York, con exclusión casi total de la hormonoterapia. Sostiene el autor que estas drogas mejorarían notablemente el estado de mayor fragilidad capilar que se presenta en la embarazada, y por este mecanismo podrían evitar el aborto. Este concepto, emitido desde 1943, tiene como seguidores a quienes han podido obtener con él resultados satisfactorios. No es de universal aceptación, y sus bases fisiopatológicas no son más firmes que las de otros tratamientos ya enunciados.

\section{MEDIDAS GENERALES}

Sean cuales fueren los sistemas de terapéutica medicamentosa que se empleen en el tratamiento de la amenaza de aborto, la paciente se beneficiará enormemente de la práctica de ciertas medidas de orden higiénico y sicoterápico que están consagradas por el uso y que siguen siendo en definitiva, la verdadera base del tratamiento preventivo del aborto.

El reposo en cama es de aceptación universal. Aún los más pesimistas, los que piensan que la amenaza de aborto no necesita tratamiento pues los huevos que fatalmente han de ser expulsados lo serán a pesar de todos los esfuerzos terapéuticos, y los que no han de ser expulsados completarán su desarrollo aunque nada se haga por ayudarlos, aceptan que en el caso de la amenaza de aborto el reposo de la paciente es útil, y dan generalmente una prueba de tres días de reposo, acompañados de sedación con feno- 
barbital, para luego permitir que la paciente continúe su vida normal y el embarazo siga la evolución a que la fatalidad lo ha condenado. El reposo en cama sigue siendo, pues, un procedimiento que no ha perdido nada de su importancia.

La abstinencia sexual absoluta es otra medida de aceptación universal que deberá acompañar siempre a cualquier procedimiento terapéutico.

La sedación del sistema nervioso y el uso de analgésicos son también de capital importancia. Repetimos que no nos parece aconsejable el uso de drogas que tengan alto poder antiespasmódico, por los inconvenientes anotados atrás. La droga más ampliamente utilizada hoy en día para llenar esta necesidad, es el fenobarbital, acompañado o no del uso de tranquilizadores y ataráxicos, según las necesidades.

En fin, el soporte sicológico adecuado para la paciente que amenaza abortar se tiene hoy en día como un elemento de importancia capital. El avance y la actualidad de la medicina sicosomática no han dejado de lado el problema del aborto, y así este factor se tiene hoy en día como fundamental en la etiología del aborto aislado o de repetición. De manera que el obstetra o ginecólogo debe obrar siempre como un activo sicoterapeuta, tratando de llevar su influencia no solamente a la paciente sino a todo el medio familiar que la rodea.

El uso de todas estas medidas generales e inespecíficas será en el tratamiento de la amenaza de aborto un elemento tan valioso o más que la prescripción medicamentosa.

\section{CONCLUSION}

Nos damos perfecta cuenta de que después de estas resumidas apreciaciones sobre terapéutica anti-abortiva, se llegaría a la conclusión de que la amenaza de aborto no tiene tratamiento racional, o de que el autor quiere llevarlos a un desesperado nihilismo terapéutico. No se trata de influír en el ánimo de los lectores, para seguir una conducta derrotista. Se ha hecho simplemente un análisis imparcial de los sistemas terapéuticos y de la falta de bases fisiopatológicas concretas de casi todos ellos, para 
concluír que el problema del aborto es probablemente el más serio y de mayores consecuencias sociales que afronta la especialidad, tanto que justifica plenamente las palabras de Carl T. Javert, cuando dedica su libro a "los millones de hombres, mujeres y niños que escaparon al sino de un aborto en su primera lucha por la existencia (in útero), y que están actualmente empeñados en su segunda lucha por la vida (ex útero) la cual, aún con los riesgos adicionales de la guerra, el matrimonio, el embarazo, el accidente y la enfermedad, es mucho menos peligrosa".

Sean cuales fueren las predilecciones terapéuticas de cada uno, no olvidemos que la paciente debe recibir un soporte sicológico lo más eficiente posible, y que las medidas generales enunciadas deben tener predilección en el tratamiento de la amenaza de aborto. Nadie podría decirnos si después de una adecuada preparación sicológica, cualquiera de las medicaciones que empleemos más o merıos empíricamente, no va a obrar como un elemento sicoterápico de primer orden.

\section{b) TRATAMIENTO DEL ABORTO INEVITABLE}

Son en general muy pocos los problemas de tratamiento que plantea el aborto inevitable. Una vez llegada la situación a la ruptura del saco ovular o a la dilatación del cuello uterino con comienzo de expulsión del contenido hacia la vagina, la labor terapéutica debe encaminarse a procurar la pronta evacuación del útero por medio del empleo de ocitócicos, a la prescripción de antibióticos si se consideran indicados, y a la vigilancia estricta de la paciente.

No creemos que la condición de aborto inevitable sea indicación perentoria de evacuación quirúrgica del útero, salvo en los casos en que, antes de expulsarse el huevo, se presente una hemorragia considerable que obligue a terminar el episodio. Son muy numerosos los casos en que con el empleo de ocitócicos se completa satisfactoriamente el aborto, sin necesidad de nuevas intervenciones. 
Es natural que una vez expulsado el huevo, la paciente debe seguir bajo vigilancia estricta para estar seguros de que el aborto espontáneo ha sido en realidad completo, y que no se está en presencia de un aborto incompleto que requerirá una conducta más activa.

\section{c) TRATAMIENTO DEL ABORTO INCOMPLETO}

Cuando se ha producido la expulsión del embrión o feto en los casos en que éste existe, y de uno o varios fragmentos de placenta, en los embarazos de menos de quince semanas es lo más frecuente que permanezcan adheridos a la pared uterina restos placentarios que estarán impidiendo la buena retracción del útero, y por consiguiente manteniendo el estado de hemorragia. El uso de ocitócicos en estos casos rara vez se verá coronado por el éxito, pues cuando las contracciones uterinas que ocasionaron el aborto no han sido suficientes para desprender y expulsar la totalidad de la placenta, es difícil que las contracciones producidas por los ocitócicos puedan hacerlo.

Esta circunstancia hace que en el aborto incompleto se haga más precisa la indicación de la evacuación quirúrgica del útero por medio del raspado y extracción de los restos ovulares remanentes. Esta indicación la damos basados en que si el aborto espontáneo no se hace de una vez completo y pasa a ser aborto incompleto, es muy improbable que su evolución espontánea lo lleve a completarse, y en los pocos casos en que esto ocurre, se hará al precio de gran pérdida de sangre para la paciente y de un alto riesgo de infección.

La evacuación de la cavidad uterina se hará bajo anestesia general o raquídea. En la casi totalidad de los verdaderos abortos incompletos es innecesaria la dilatación artificial del cuello, pues en casi todos los casos éste es suficientemente permeable para pasar una pinza de falsos gérmenes o üna cureta roma. No es inoperante recalcar una vez más sobre los cuidados que deben te. nerse durante el raspado, por el peligro de perforaciones de la pared uterina. 
En la época actual se ha estado abandonando casi por completo la práctica del taponamiento intrauterino después del raspado, pues la experiencia ha demostrado que es innecesario, y no siendo indispensable, no vale la pena agregar dentro del útero un cuerpo extraño, que representa una manipulación adicional y un mayor riesgo de infección.

En el manejo del aborto incompleto deben tenerse también muy en cuenta las condiciones generales de la paciente, la cantidad de sangre perdida, la intensidad de la hemorragia actual, la presencia o ausencia de estado de shock, etc., y atender con las medidas adecuadas (transfusión, antibióticos, etc.) a todas estas situaciones.

\section{d) TRATAMIENTO DEL ABORTO SEPTICO}

El aborto séptico es usualmente un aborto incompleto al cual se agrega la sepsis como complicación. Como aborto incompleto complicado, exige la evacuación quirúrgica del útero. En el tratamiento del aborto séptico ha existido siempre un dilema difícil de resolver y que consiste en si debe tratarse primero la sepsis, es decir enfriar el caso, o tratarse primero el aborto, es decir practicar el raspado. Creemos que no se debe adherir completamente a ninguna de las dos tendencias, sino que se debe tratar con prelación la condición más urgente. Así, cuando domina claramente el cuadro séptico, y la paciente no tiene una hemorragia severa, será preferible tratar primero la sepsis, y luego evacuar el útero cuando los signos de infección hayan cedido. En cambio, si se está ante el caso del aborto séptico y al mismo tiempo hay una hemorragia profusa, creemos que debe procederse al raspado inmediatamente, sin olvidar, como es obvio, comenzar al mismo tiempo el tratamiento de la infección.

Debemos recalcar especialmente sobre el hecho de que en los casos sépticos el raspado debe ser especialmente cuidadoso, pues cuando hay ya establecido un estado de miometritis la pared uterina se hace eminentemente friable y los riesgos de perforación aumentan extraordinariamente. 


\section{d) TRATAMIENTO DEL ABORTO RETENIDO}

Mientras no se presente hemorragia, el aborto retenido no exige tratamiento inmediato, pues la retención del producto de la concepción in útero no representa en realidad un riesgo especial para la madre. Pero aún siendo así, muchas veces ocurre que las pacientes van adquiriendo un estado de verdadero problema sicológico cuando saben que son portadores de un huevo muerto, y llegan a exigir en forma perentoria la solución de su situación.

Cuando llega el caso de tener que terminar un aborto retenido, sea por exigencia de la paciente, sea porque comienza a presentarse un estado de hemorragia más o menos constante, aunque no en realidad muy abundante, como suele ser, se puede recurrir al uso de ocitócicos con el objeto de intentar un aborto espontáneo. Esta medicación fracasa en la mayoría de las veces, y es raro que no tenga que terminarse el caso por dilatación y raspado. 